\title{
TEM and In-situ Liquid Cell Characterization of Copper Nanowire Growth Mechanisms
}

\author{
A.R. Rathmell,* R.R. Unocic, ${ }^{* *}$ J.Y. Howe, ${ }^{* *}$ and B.J. Wiley* \\ * Department of Chemistry, Duke University, Durham, NC 27708 \\ ** Materials Science \& Technology Div., Oak Ridge National Laboratory, Oak Ridge, TN 37831
}

Copper nanowires (CuNWs) can be painted onto a surface to create an inexpensive, flexible and transparent conductor for use in smart windows, organic light emitting diodes, and thin-film solar cells. Transparent conductors based on CuNWs are potentially less expensive than the industry standard, indium tin oxide (ITO), because CuNWs can be deposited from solution at coating rates up to 1000 times faster than vapor-deposited ITO. Recent work has demonstrated the feasibility of synthesizing large quantities of CuNWs by reducing $\mathrm{Cu}\left(\mathrm{NO}_{3}\right)_{2}$ with $\mathrm{N}_{2} \mathrm{H}_{4}$ in an aqueous solution containing $\mathrm{NaOH}$ and $\mathrm{C}_{2} \mathrm{H}_{4}\left(\mathrm{NH}_{2}\right)_{2}$ [1]. By taking samples from the reaction at different times for imaging in a SEM, we have previously observed spherical copper seed crystals form in the early stages of the reaction, after which CuNWs sprout and grow from those seeds [1]. Fig. 1a shows a TEM image of a wire that grew from a spherical seed. It is apparent from diffraction contrast TEM imaging that the seed crystals contain multiple defects, including stacking faults and twins, as shown in Fig. 1b. The CuNWs themselves have a [ 110$]$ growth orientation and also contain twins (Fig. 1c). The inset high-resolution TEM image shows twins present along the (l $\left.\begin{array}{ll}1 & 1\end{array}\right)$ plane. Although it is known that the concentration of the reagents (especially the capping agent, $\left.\mathrm{C}_{2} \mathrm{H}_{4}\left(\mathrm{NH}_{2}\right)_{2}\right)$ and the reaction temperature affect the dimensions of the nanowires, it is not yet clear what driving force causes copper atoms to assemble anisotropically from the surface of the seed to form nanowires. The growth of Pt nanowires (PtNWs) from large, spherical aggregates of nanoparticles is somewhat similar in that both nanowires grow from spherical nanoparticles [2]. The growth mechanisms differ in that hundreds of PtNWs grow from spherical aggregates in a structure similar to a sea urchin, where in the case of CuNWs, only one or two wires typically grow from a single seed.

To provide further insight into the nucleation and growth mechanisms of CuNWs, direct observations of $\mathrm{CuNW}$ growth was studied using an in-situ liquid flow cell TEM holder (Hummingbird Scientific) within a Hitachi HF3300 S/TEM operated at 300kV. The liquid flow cell holder assembly consists of an integrated fluid supply and return lines that allows for the injection of liquid (via a microfluidic syringe pump and microfluidic tubing) to a microfluidic cell (created by stacking two silicon microchips with 50-nm-thick electron transparent silicon nitride viewing

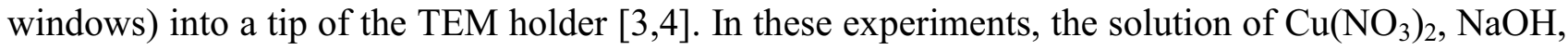
$\mathrm{C}_{2} \mathrm{H}_{4}\left(\mathrm{NH}_{2}\right)_{2}$ and $\mathrm{N}_{2} \mathrm{H}_{4}$ was heated at $70{ }^{\circ} \mathrm{C}$ for 5 minutes. The solution was then drawn into a glass syringe and immediately delivered to the microfluidic cell at a flow rate of $5 \mu \mathrm{L} / \mathrm{min}$. The precipitation of the copper seed crystals at the early stages of the reaction is shown in Fig $2 \mathrm{a}$. The subsequent growth of the CuNWs from the seed crystals is shown in Fig. 2b. Through real-time observations of nanowire synthesis in the liquid phase, it is possible to provide the experimental observations necessary for a predictive theory of nanostructure growth, and therefore lay the foundation for the future design and production of nanostructured materials with tailored electrical, thermal, mechanical, optical, and chemical properties [5]. 


\section{References}

[1] A.R. Rathmell et al., Advanced Materials, 22 (2010) 3558.

[2] J. Chen et al., J. Am. Chem. Soc. 126 (2004) 10854.

[3] N. de Jonge et al., Proc Nat Acad Sci, 106, 7 (2009) 2159.

[4] E.A. Ring et al., Microscopy and Microanalysis, 16 (2010) 622.

[5] Research supported by ORNL's Shared Research Equipment (SHaRE) User Facility, which is sponsored by the Office of Basic Energy Sciences, U.S. Department of Energy. BJW was supported with start-up funds from Duke University.
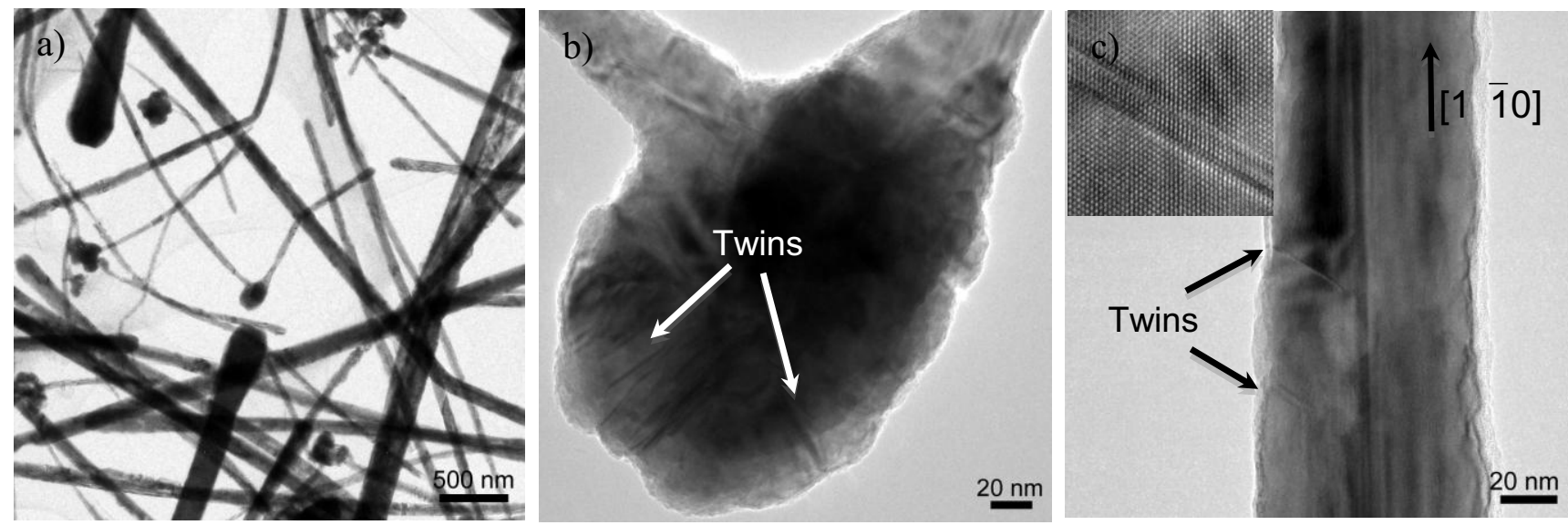

FIG 1. a) Bright field TEM image of CuNWs synthesized by reducing $\mathrm{Cu}\left(\mathrm{NO}_{3}\right)_{2}$ with $\mathrm{N}_{2} \mathrm{H}_{4}$ in an aqueous solution of $\mathrm{NaOH}$ and $\mathrm{C}_{2} \mathrm{H}_{4}\left(\mathrm{NH}_{2}\right)_{2}$. b) $\mathrm{Cu}$ seed crystal with multiple twins. c) Individual CuNW with a $\left[\begin{array}{ll}1 & 1\end{array}\right]$ growth orientation (inset HRTEM image showing twins present along $\left(\begin{array}{ll}1 & \overline{1} 1\end{array}\right)$ planes).
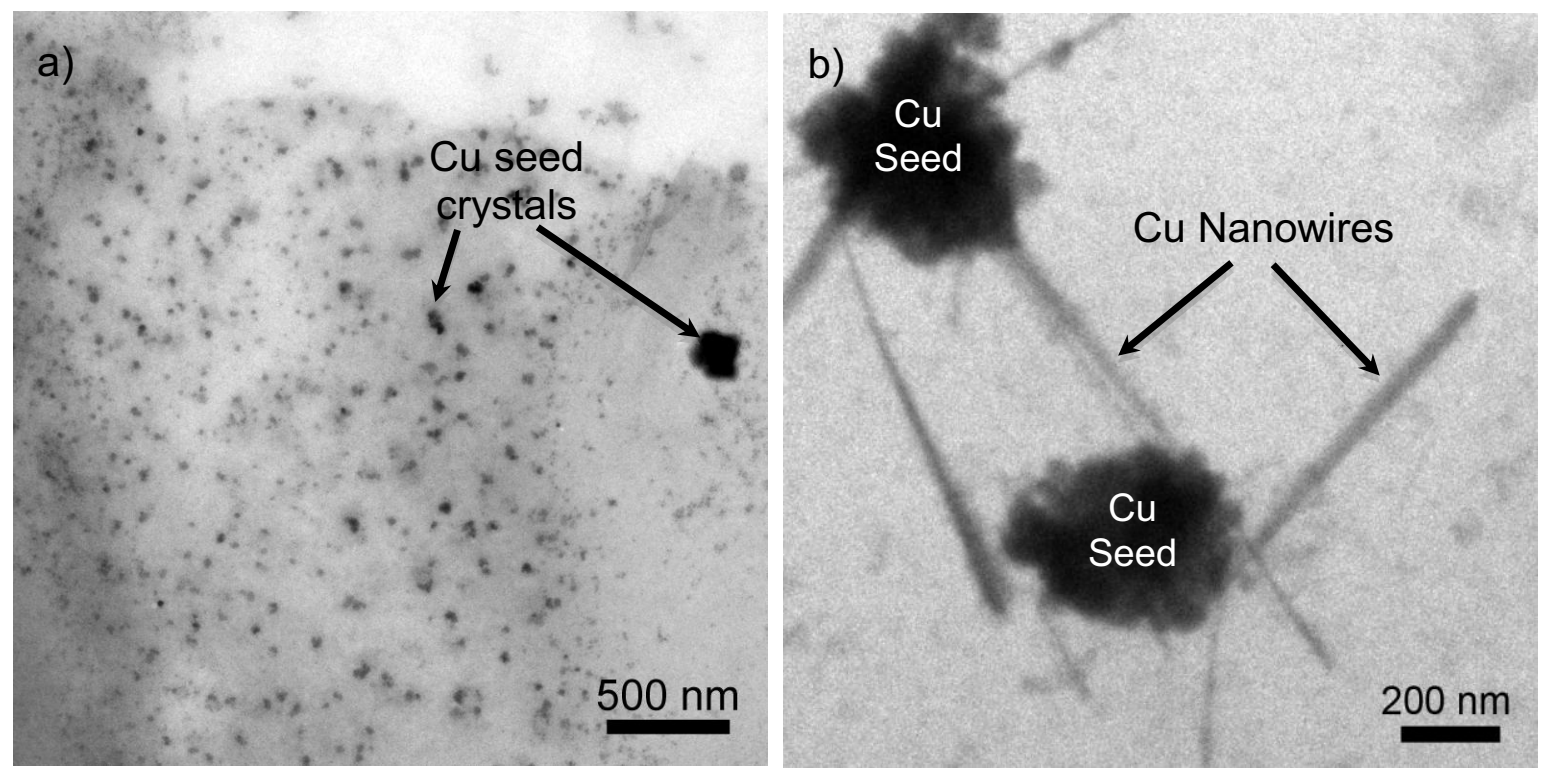

FIG 2. Bright-field TEM images acquired from an in-situ liquid cell TEM experiment showing the a) nucleation of copper seed crystals during the early stages of CuNW growth and b) the subsequent growth of $\mathrm{CuNWs}$ from the $\mathrm{Cu}$ seed crystals. 